This is a preprint of the accepted article: Foerster, R. M. \& Schneider, W. X. (2015). Anticipatory eye movements in sensorimotor actions: On the role of guiding fixations during learning. Cognitive Processing, 701, 1-5. doi: 10.1007/s10339-015-0701-1. The final publication is available at http://link.springer.com/article/10.1007/s10339-015-0701-1/fulltext.html

\title{
Anticipatory eye movements in sensorimotor actions: On the role of guiding fixations during learning
}

\author{
Rebecca M. Foerster ${ }^{1,2}$, \& Werner X. Schneider ${ }^{1,2}$ \\ ${ }^{1}$ Department of Psychology and ${ }^{2}$ Cluster of Excellence 'Cognitive Interaction Technology', Bielefeld Uni- \\ versity, Germany
}

Correspondence to

Rebecca M. Foerster

Department of Psychology, Bielefeld University

Cognitive Interaction Technology - Center of Excellence (CITEC), Bielefeld University

P. O. Box 100131, D-33501 Bielefeld, Germany

Phone: 011-49-106-4503

Fax: 011-49-106-156934

E-mail rebecca.foerster@uni-bielefeld.de

\begin{abstract}
During object-based sensorimotor tasks, humans look at target locations for subsequent hand actions. These anticipatory eye movements or guiding fixations seem to be necessary for a successful performance. By practicing such a sensorimotor task, humans become faster and perform fewer guiding fixations (Foerster and Schneider In Prep; Foerster et al. 2011). We aimed at clarifying whether this decrease in guiding fixations is the cause or effect of faster task completion time. Participants may learn to use less visual input (fewer fixations) allowing shorter completion times. Alternatively, participants may speed up their hand movements (e.g., more efficient motor control) leaving less time for visual intake. The latter would imply that the number of fixations is directly connected to task speed. We investigated the relationship between the number of fixations and task speed in a computerized version of the number connection task (Foerster and Schneider 2015). Eye movements were recorded while participants clicked in ascending order on 9 numbered circles. In 90 learning trials, they clicked the sequence with a constant spatial configuration as fast as possible. In the subsequent experimental phase, they should perform 30 trials again under high-speed instruction and 30 trials under slow-speed instruction. During slow-speed instruction, fixation rates were lower with longer fixation durations and more fixations were performed than during high-speed instruction. The results suggest that the number of fixations depends on both the need for visual intake and task completion time. It seems that the decrease in anticipatory eye movements through sensorimotor learning is at the same time a result and a cause of faster task performance.
\end{abstract}

Keywords anticipatory eye movements, guiding fixations, sensorimotor learning, fixation rate

\section{Introduction}

Sensorimotor actions are accompanied by anticipatory eye movements, i.e. humans fixate upcoming target locations before they act on them (Epelboim et al. 1995; Land et al. 1999; Hayhoe et al. 2003; Land and Tatler 2009; Foerster et al. 2011). Before grasping a pen, for instance, we look at the pen at exactly that position where the hand will contact it. It has been shown that these anticipatory guiding fixations are necessary to perform accurately (Prablanc et al. 1979; Prablanc et al. 1986; Abrams et al. 1990).

Besides guiding fixations (Epelboim et al. 1995; Land and Tatler 2009), there are two further types of fixations that are performed during object-related sensorimotor actions. Searching fixations are used to find the upcoming target object (Foerster and Schneider In Prep; Epelboim et al. 1995; Foerster and Schneider 2015). Checking fixations are used to determine the status of a completed target object (Land and Tatler 2009; Foerster and Schneider 2015). When practicing a sensorimotor task, performance does not only speedup, fewer fixations are also performed (Epelboim et al. 1995; Sailer et al. 2005; Foerster et al. 2011) - of all types (Foerster and Schneider In Prep). 
There are two possibilities why the number of fixations decreases with increasing task expertise. On the one hand, performers may learn how to execute the action with less visual information. The ability to manage the task with less visual intake allows for omitting fixations. As a fixation needs time to be planned and executed, time is saved. Thus, fixation saving may be the reason why a performer can speed-up at all. On the other hand, the number of fixations executed might be obligatorily connected to task completion time. Given a guiding fixation needs $300 \mathrm{~ms}$, then about 3 guiding fixations can be executed per second. When there is less time, fewer fixations have to be performed if fixation duration and rate shall be held constant. Thus, under the assumption of obligatory coupling between the number of fixations and time available, a decrease in the number of fixations takes place whenever there is a task speed-up. The reason for the performance speed-up over time could, for instance, be caused by improved hand motor control. Thus, even if the speedup in performance has nothing to do with the visual intake needed, a decrease in the number of fixations with learning should take place.

We aimed at clarifying whether the decrease of fixations is the cause or effect of performance speed-up in an object-based sensorimotor task over the course of learning. To reveal the relationship between the number of fixations and task completion time, we recorded eye movements of participants performing a computerized version of the number connection test or trial making test A (Foerster and Schneider In Prep; Foerster and Schneider 2015). In this object-based sensorimotor sequence task, participants had to click on 9 numbered circles in ascending order. In 90-learning trials, they clicked the same sequence as fast as possible. In the subsequent experimental phase, they performed 30 trials again under high-speed instruction and 30 trials under slow-speed instruction. If the number of fixations is obligatorily connected to task completion time, then fixation rate and duration should stay constant across speed conditions, while more fixations should be performed under slow-speed instruction. If the amount of visual intake is connected to the amount of visual information needed to complete the task, then the number of fixations should stay constant across speed conditions, while fixation rate should be lower and fixation duration longer under slow-speed instruction.

\section{Methods}

\section{Participants}

Fourteen right-handed students ( $M=26.07$ years, 5 male) participated in the experiment. Participants had normal or corrected-to-normal visual acuity, were naïve with respect to the study's purpose, and were paid for participation.

\section{Apparatus and Stimuli}

Stimuli were displayed on a 19-in. color monitor (1,024x768 pixels, $100 \mathrm{~Hz})$. The keyboard, the computer mouse, and an extra-large mouse pad $(88 \times 32 \mathrm{~cm})$ were used. Participants' right gaze position was recorded every millisecond (EyeLink 1000 tower system, SR Research). Participants' heads were fixed at a viewing distance of $71 \mathrm{~cm}$. The Experiment Builder software (SR Research) controlled the experimental procedure. A black dot served as mouse cursor (diameter $=.43^{\circ} \mathrm{v}$.a.). Target stimuli consisted of 9 numbered unfilled circles with constant spatial arrangement (black bold Arial, font size 35, $2.04^{\circ} \mathrm{v}$.a diameter) displayed on a gray background. Circle 1 was located in the center of the screen. The spatial distribution of circles 2-8 was randomly generated with the prerequisite that the outer fields of an imagined 3x3 grid contained one circle and circles were minimally $2.04^{\circ} \mathrm{v}$.a. apart from each other and from the screen border. The generated spatial configuration was used throughout the experiment (Figure 1).

\section{Procedure}

The experiment was divided into one learning phase and two testing phases (counterbalanced). Each phase started with a written instruction on the screen followed by a 9-point calibration and validation procedure (validation accuracy $<1.0^{\circ}$ v.a.). During the 90 -trial learning phase, participants clicked as fast as possible in ascending order on the 9 numbered circles (high-speed instruction). An example trial preceded the phase. The high-speed instruction was also applied in one of the 30-trial testing phases. In the other testing phase, participants were instructed to complete the same clicking sequence with an indicated constant trial completion time, while executing still continuous movements (slow-speed instruction). The instructed slow completion time was individually chosen by doubling the mean completion time of a participant's last 30-learning trials. A click was counted as correct, if the cursor was within a diameter of $3.06^{\circ} \mathrm{v}$.a. around the current target's center. A correct click was followed by a high-pitched tone. After having clicked the sequence, a feedback display indicated trial completion time. During slow-speed instruction, the instructed time and de- 
viation to it were displayed additionally. After every 10 trials, the number of completed and total blocks was indicated. Participants started each of the 15 blocks and 150 trials by pressing the space bar. The best participant of a phase (fastest, least deviation) earned $5 €$ extra.

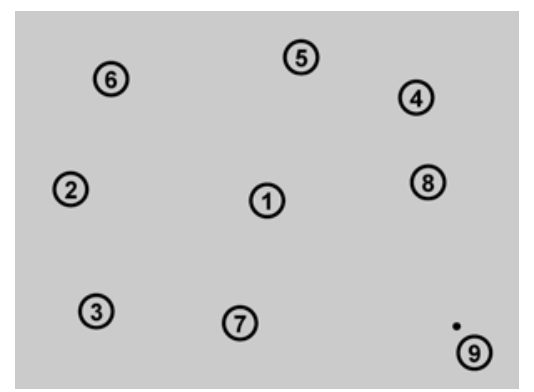

Figure 1. Display during sequential-clicking task throughout the experiment.

\section{Analysis}

Fixations were detected by the SR Research's default algorithm (not a blink and velocity $<30 \%$ or acceleration $<8000^{\circ} / \mathrm{s}^{2}$ ). Number, duration, and rate of fixations and fixation types (guiding, searching, checking) were compared across slow-speed and high-speed conditions with paired $t$-tests. Fixation types were defined according to their landing positions (Foerster and Schneider 2015): Fixations on an upcoming target as searching, fixations on a current target as guiding, and fixations on a previous target as checking type (interest area of $3.27^{\circ} \mathrm{v} . \mathrm{a}$. diameter).

\section{Results}

With practice, trial completion times and the number of fixations decreased as is typical for sensorimotor learning (linear trends: time: $p<.001$; fixations: $p<.001$ ). However, fixation rate decreased (linear trend $p<.05$ ), and fixation duration increased (linear trend $p<.05$ ). This is a first hint that the number of fixations is not obligatorily connected to trial completion time. With learning, the number of fixations decreased more than would have been predicted by the clicking speed-up. Further effects of learning the clicking task are reported in Foerster and Schneider 2015 and Foerster and Schneider In Prep.

To test whether our manipulation was successful, we compared trial completion times across high-speed and slow-speed instructions (Figure 1a). Participants were significantly faster under high-speed (4.70s) than under slow-speed (9.32) instruction $(t(13)=17.32, p<.001)$. To finally answer the question whether the number of fixations is obligatorily connected to trial completion time with constant fixation durations and fixation rates, we compared the variables across slow-speed and high-speed test phases. Participants performed significantly more fixations during slow-speed (18.89) than fast-speed (12.37) testing (Figure 1a, $t(13)=9.54$, $p<.001$ ), supporting a relation between the number of fixations and trial completion time. However, fixation rates and durations were not constant across phases (Figure 1b). Under slow-speed instruction, fixation rate was significantly lower and fixation duration was significantly longer than under high-speed instruction (rate: $2.06 / \mathrm{s}$ vs. $2.67 / \mathrm{s}, t(13)=6.17, p<.001$; duration: $455.18 \mathrm{~ms}$ vs. $350.59 \mathrm{~ms}, t(13)=4.41, p<.001$ ). Therefore, the number of fixations did not increase proportionally to performance speed-up, but remained behind that. The additional time was filled by longer fixation durations.

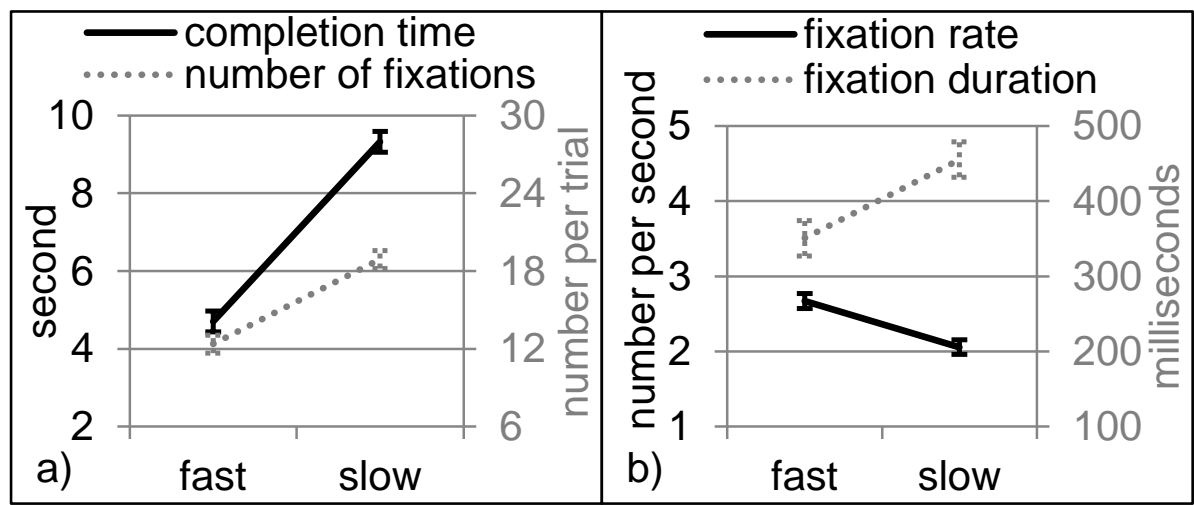

Figure 2. a) Trial completion time and the number of fixations and b) fixation rate and fixation duration across fast-speed and slow-speed testing. Error bars represent standard errors of the mean of the paired differences across speed conditions. 
To reveal which fixation types were responsible for the result pattern, we compared number, rate, and duration individually per guiding, searching, and checking types. The analyses revealed that the anticipatory guiding fixations were mainly responsible for the overall result pattern (Table 1, Figure 2). Guiding fixations increased in number, while their rate decreased, and their duration increased under slow-speed compared to high-speed instruction. Number and duration of searching fixations, however, did not change with speedinstruction, while searching rate was lower with slower compared to faster clicking. Checking fixations increased in number and rate under slow-speed compared to high-speed instruction, while their duration was not affected.

Table 1. Statistics of paired $t$-tests for number, rate, and duration of guiding, searching, and checking fixations across fast-speed and slow-speed testing.

\begin{tabular}{|c|c|c|c|}
\hline fixations & guiding & searching & checking \\
\hline number & $t(13)=8.92, p<.001$ & $t(13)=.86, p=.40$ & $t(13)=3.13, p<.01$ \\
\hline rate & $t(13)=4.22, p<.01$ & $t(13)=2.58, p<.05$ & $t(13)=2.48, p<.05$ \\
\hline duration & $t(13)=5.52, p<.001$ & $t(13)=.46, p=.65$ & $t(12)=.13, p=.90$ \\
\hline
\end{tabular}

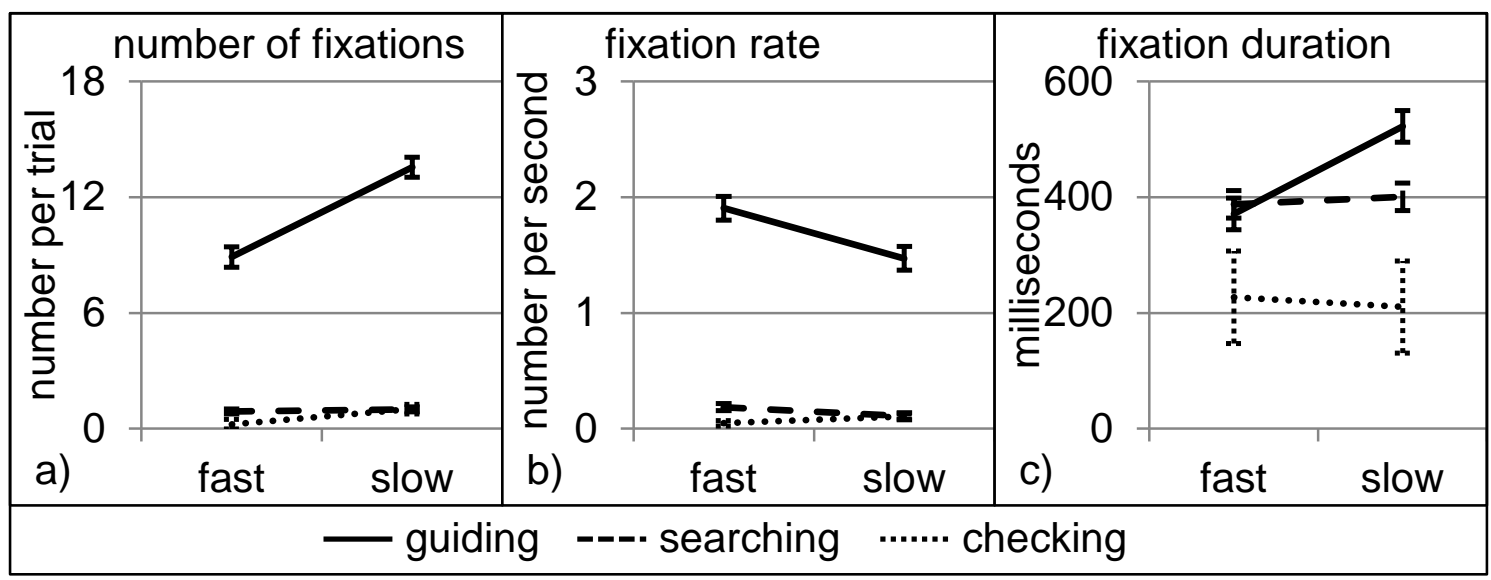

Figure 3. a) Number, b) rate, and c) duration of guiding, searching, and checking fixations across fast-speed and slow-speed testing. Error bars represent standard errors of the mean of the paired differences across speed conditions.

\section{Discussion}

We aimed at clarifying whether the decrease in fixations observed through sensorimotor learning is the cause or the effect of the task speed-up. Learning to save fixations and thus time might be the reason why higher expertise leads to faster performance. Alternatively, performance speed-up may have nothing to do with the amount of visual intake required. Instead, this gain in speed could be based on hand motor control improvements. A shorter task completion time should in turn leave less time for visual intake given a fixation needs a specific duration. In this case of direct coupling between the number of fixations and task completion time, constant fixation rates and durations are expected across different task-speeds.

We investigated whether the number of fixations is exclusively modulated by task-speed in a computerized version of the number connection test (Foerster and Schneider 2015). In this object-based sensorimotor task, participants click on 9 numbered circles in ascending order. During 90-learning trials, participants clicked the sequence as fast as possible. In subsequent 60-testing trials, this high-speed instruction was given in one half of the trials and a slow-speed instruction in the other half.

Results revealed that the number of fixations was higher during slow than during fast task completion, arguing for an upscaling of visual intake with available time. However, fixation rate was lower under slow-speed than under high-speed instruction. Thus, the upscaling in the number of fixations was not proportional to the task speed-up, but remained behind that. The remaining time was filled by longer fixation durations. The anticipatory guiding fixation type produced this result pattern. Results argue for a relationship between the number of fixations and the amount of visual intake needed to complete the task successfully. With higher task expertise, less visual intake should be required, resulting in fewer fixations per second. Thus, it is plausible that performance speed-up when practicing object-based sensorimotor actions is partly achieved by learning to omit visual intake. First, performers might learn which visual information is not obligatorily necessary to complete the task successfully and do no longer gather this information. Second, performers may improve in accuracy when saccading to upcoming target locations from long-term memory (Foerster et al. 
2011; Foerster et al. 2012), so that they can omit corrective saccades. Thereby, they end up with the minimum of guiding fixations needed. This reasoning would also explain why fixation rate decreases over the course of high-speed learning (here and in Foerster and Schneider 2015).

In sum, the present study revealed that the number of fixations in object-based sensorimotor tasks is not in a one-to-one manner connected to task completion time. Instead, expertise also modulates the number of executed fixations by determining the amount of visual intake required to perform the task successfully. Probably, the number of fixations depends partly on the need for visual intake and partly on task completion time. Therefore, the decrease in anticipatory eye movements observed through sensorimotor learning seems to be at the same time a cause and a consequence of faster task performance.

\section{Acknowledgements}

This research was supported by the Cluster of Excellence Cognitive Interaction Technology 'CITEC' (EXC 277) at Bielefeld University, which is funded by the German Research Foundation (DFG).

\section{References}

Abrams RA, Meyer DE, Kornblum S (1990) Eye-hand coordination: Oculomotor control in rapid aimed limb movements. J Exp Psychol Hum Percept Perform 16:248-267.

Epelboim JL, Steinman RM, Kowler E, et al. (1995) The function of visual search and memory in sequential looking tasks. Vision Res 35:3401-3422.

Foerster RM, Carbone E, Koesling H, Schneider WX (2011) Saccadic eye movements in a high-speed bimanual stacking task: Changes of attentional control during learning and automatization. J Vis 11(7):9:1-16. doi: 10.1167/11.7.9

Foerster RM, Carbone E, Koesling H, Schneider WX (2012) Saccadic eye movements in the dark while performing an automatized sequential high-speed sensorimotor task. J Vis 12(2):8:1-15. doi: $10.1167 / 12.2 .8$

Foerster RM, Schneider WX Saccades to invisible manual action targets: Long-term memory-based attention in the number connection test. Manuscr. Prep.

Foerster RM, Schneider WX (2015) Expectation violations in sensorimotor sequences: Shifting from LTMbased attentional selection to visual search. Ann N Y Acad Sci. doi: 10.1111/nyas.12729

Hayhoe MM, Shrivastava A, Mruczek R, Pelz JB (2003) Visual memory and motor planning in a natural task. J Vis 3:49-63. doi: DOI 10:1167/3.1.6

Land M, Mennie N, Rusted J (1999) The roles of vision and eye movements in the control of activities of daily living. Perception 28:1311-1328. doi: 10.1068/p2935

Land MF, Tatler BW (2009) Looking and acting. Oxford University Press, New York

Prablanc C, Echallier JF, Komilis E, Jeannerod M (1979) Optimal response of eye and hand motor systems in pointing at a visual target. Biol Cybern 35:113-124.

Prablanc C, Pellison D, Goodale MA (1986) Visual control of reaching movements without vision of the limb. Exp Brain Res 62:293-302.

Sailer U, Flanagan JR, Johansson RS (2005) Eye-hand coordination during learning of a novel visuomotor task. J Neurosci 25:8833-8842. doi: 10.1523/JNEUROSCI.2658-05.2005 